\title{
A comprehensive model for battery State of Charge prediction
}

\author{
Bart Homan, Gerard J.M. Smit \\ Computer Architectures and Embedded Systems \\ University of Twente \\ Enschede, the Netherlands \\ b.homan@utwente.nl
}

\author{
Richard P. van Leeuwen \\ Chair renewable energy \\ Saxion University of Applied Sciences \\ Enschede, the Netherlands \\ r.p.vanleeuwen@saxion.nl
}

\author{
Marnix V. ten Kortenaar \\ Dr Ten B.V. \\ Wezep, the Netherlands
}

\begin{abstract}
In this paper the relatively simple model for State of Charge prediction, based on energy conservation, introduced in [1] is improved and verified. The model as introduced in [1] is verified for Pb-acid, Li-ion and Seasalt batteries. The model is further improved to accommodate the rate capacity effect and the capacity recovery effect, the improvements are verified with lead-acid batteries. For further verification the model is applied on a realistic situation and compared to measurements on the behavior of a real battery in that situation. Furthermore the results are compared to results of the well-established KiBaM model. Predictions on the $\mathrm{SoC}$ over time done using the proposed model closely follow the SoC over time calculated from measured data.
\end{abstract}

The resulting improved model is both simple and effective, making it specially useful as part of smart control, and energy usage simulations.

Index Terms-Storage, Predictive model, Smart grid, Energy management

\section{INTRODUCTION}

Batteries are an important part of everyday energy usage. Examples include using a battery for black-out situations, using a battery to store electricity generated by pv-panels during the day and for usage during the night, and charging an electric vehicle (EV). Simulations are used for example to predict weak points in existing grids [2] or to explore the possibilities of new types of grids [3]. To accurately simulate the energy usage in a grid, accurate models are needed for all devices connected to the grid. There are many models available that describe the behavior of batteries [4], [5]. Some of the models, like the Dualfoil model [6] and the kinetic battery model (KiBaM) [7] are applicable for only one type of battery. While other models, like the Coulomb counting model [8] are useful for various types of batteries. Some of these models are rather comprehensive and complicated, and require intimate knowledge of the battery. The Dualfoil model for instance is generally accepted as an excellent model for Li-ion batteries, but it requires over 50 input parameters, e.g. the thickness of the separator and the porosity of the cathode, information that is not readily available for each battery. The Coulomb counting model on the other hand is rather simple and requires only few input parameters, but generally yields a too ideal representation of battery behavior. So some of the available models are too complex to be used in energy-grid simulations, while other models are simple but not accurate enough.

A simple but effective model was originally developed for thermal storage but can be applied for electric storage as well [1], [9]. Using this model it is possible to predict the amount of energy that can be discharged from a battery, or charged into a battery at any given state of charge (SoC). This model had some drawbacks, however, for instance the capacity recovery effect and rate capacity effect are not covered. In this paper, an extension of the model for battery SoC prediction, presented in [1] is developed.

\section{Methods}

\section{A. Basic predictive battery SoC model}

The model to determine the SoC of a battery during discharging and charging, proposed in [1] is based on energy conservation, combined with insights from experimental data. The governing equation of the model is:

$$
\Delta S_{t}=\Delta C_{t}-\Delta D_{t}-\Delta L_{t}
$$

In which $\Delta S_{t}$ signifies the change of stored energy, $\Delta C_{t}$ the charged energy, $\Delta D_{t}$ the demand and $\Delta L_{t}$ the energy loss, all within a time interval $\Delta t$ which is the discrete time interval $(t-1, t)$.

$$
\Delta D_{t}=\Delta t \cdot P_{e, t}=\Delta t \cdot I_{t} \cdot U_{d c, t}
$$

In which $P_{e, t}$ the electric power consumption, $I_{t}$ the discharging current and $U_{d c, t}$ the voltage output of the battery, both measured at the inverter. The SoC is calculated by equation 3 .

$$
S o C_{t}=\frac{S_{t}}{S_{\max }}, 0 \leq S o C_{t} \leq 1
$$

The stored energy $S_{t}$ is determined by equation 4 and the maximum charged energy by equation 5 .

$$
\begin{gathered}
S_{t}=S_{t-1}+\Delta S_{t} \\
S_{\text {max }}=C \cdot U_{n}=\sum_{\tau} P_{c, t^{*}} \cdot \Delta t
\end{gathered}
$$

In which $C$ is the battery capacity (or battery rating) in Ah, $U_{n}$ the nominal voltage and $P_{c, t^{*}}$ the charging power for time $t^{*}$ which signifies a time interval during a charging cycle, for 
which: $0 \leq t^{*} \leq \tau$. The relation between the charging time and power is displayed in figure $1 \mathrm{a}$.

The maximum capacity $S_{\max }$ may be a function of time due to degradation of the battery. Hence, the sum of measured charging power may be more accurate in practice than the rate capacity.

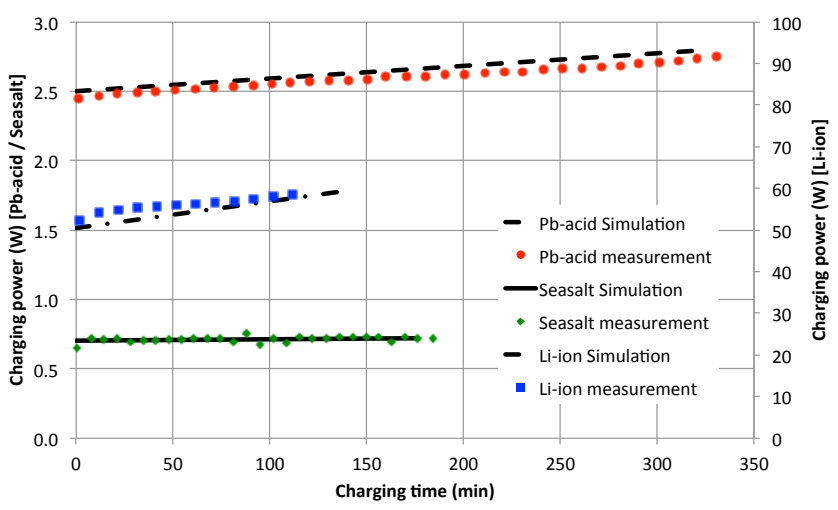

(a) Measured and predicted charging power vs charging time.

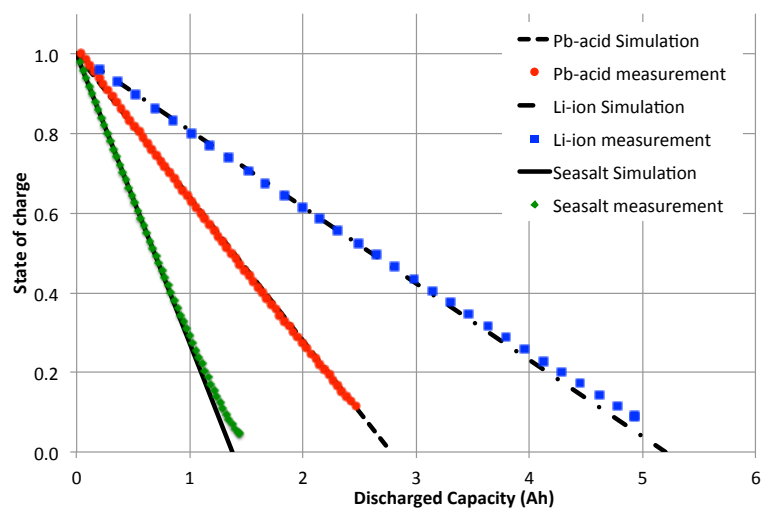

(b) Measured and predicted SoC vs discharge current

Fig. 1: Measured and predicted charging and discharging characteristics for a $\mathrm{Pb}$-acid, Li-ion and Seasalt battery. The characteristics for each battery are summarized in table I

The amount of useful energy that can be supplied by the storage is determined by $S_{\max }$ and a minimum SoC value which is determined by the minimum useful and safe to use voltage of the battery.

In [1] an average discharge current $I_{a v}$ is introduced and calculated with equation 6.

$$
I_{a v, \tau_{d c, t}}=\frac{\sum_{\tau d c, t} D_{t}}{\tau_{d c, t} \cdot U_{d c, t}}
$$

However, this equation has proven to introduce errors for discontinuous discharging processes involving various waiting periods without discharging. Also, the accuracy of the average discharge current concept in case of dynamic load variations is not yet investigated. A new and better method is developed in this paper.

The battery is usually not discharged beyond the minimum SoC, otherwise it would be damaged. In the safe region, experiments show that a linear relation exists between the decrease of the SoC and the total discharged capacity (in Ah) from the battery. This relation is displayed in figure $1 \mathrm{~b}$. For the slope, equation 7 applies.

$$
\frac{\Delta S o C}{\Delta C_{D}}=\frac{1}{C}
$$

The accuracy of the model described by equations 1-7 is proven with experiments on various battery types, described in table I, subjected to constant discharge currents.

TABLE I: Characteristics of the batteries used for verification

\begin{tabular}{llccc}
\hline Name & Type & $\mathrm{U}(\mathrm{V})$ & $\mathrm{I}_{\text {charge }}(\mathrm{mA})$ & $\mathrm{C}(\mathrm{Ah})$ \\
\hline Pb-acid & Lead acid & 6.0 & 400 & 2.7 \\
Li-ion & Lithium-ion & 25.2 & 2600 & 5.2 \\
Seasalt & Seasalt & 1.8 & 300 & 1.3 \\
\hline
\end{tabular}

The Seasalt battery is an experimental, stationary battery currently in development at the battery innovation company Dr. Ten. It is designed to be inherently safe and environmentally friendly.

\section{B. Model extension for discontinuous discharging processes}

Most batteries show two well known effects during discontinuous discharging:

- Rate capacity effect: This effect limits the recoverable $\mathrm{SoC}$ in relation to the discharging current. The higher the discharge current, the less $\mathrm{SoC}$ is recoverable from the battery.

- Capacity recovery effect: This effect occurs when a battery is discharged to a certain state, followed by a certain amount of time without discharging (the waiting period). For example, the battery can be discharged to its minimum useful voltage, but when this is followed by a waiting period, the voltage recovers and it is possible to discharge the battery further. This effect is more significant when periods of high discharge currents are followed by a waiting period, while the rate capacity effect becomes insignificant for relatively low discharging currents.

These two effects are related to each other. The capacity recovery effect is more significant when the battery is discharged with relatively high currents, i.e. when the rate capacity effect is also significant. To describe both effects, there is some analogy with a thermal storage. When a thermal storage is discharged by an outlet at the top and inlet at the bottom, cold water coming into the storage at the bottom mixes with warmer water within the storage in the bottom region. This effect is influenced by the discharge flow rate, the higher the flow rate, the more mixing will occur.

A battery can be described similarly, see figure 2. Generally, when a battery is charging, a reaction takes place at one of the electrodes (electrode 1) creating a compound in which the energy is stored (the black compound) When the battery is fully charged the compound containing the energy is present everywhere in the battery (figure 2a). When a battery is then discharged the compound containing the energy has to physically move to the other electrode (electrode 2) where 


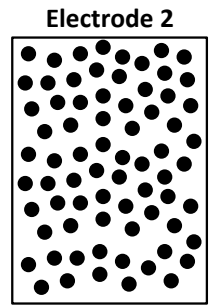

Electrode 1

(a)

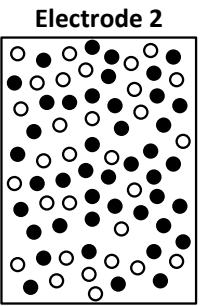

Electrode 1

(b)

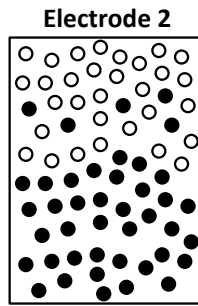

Electrode 1

(c)

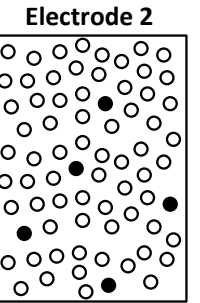

Electrode 1

(d)
Fig. 2: Schematic representation of the rate capacity effect in a battery. a) The battery is fully charged. $b$ ) The battery is discharging slowly. c) The battery is discharging fast. $d$ ) The battery is fully discharged.

another chemical reaction takes place creating another compound (the white compound) while releasing the energy. The concentration of the black compound at electrode 2 represents the voltage of the battery. So in other words if the battery is discharged, the concentration of the black compound, and thus the voltage drops until there is too little of the black compound left at electrode 2 to continue discharging, then the battery is discharged (see figure 2d).

If the battery is discharged slowly (i.e with low discharge current) the white compound is well mixed with the black compound. When $50 \%$ of the black compound has been used, (see figure $2 \mathrm{~b}$ ) the concentration of the black compound at electrode 2, and thus the voltage, is lower then when the battery is fully charged. If the battery is discharged fast (i.e with high discharge current) the black compound is used faster than the mixing occurs (see figure 2c), creating a layer of the white compound that limits access to electrode 2 for the black compound. When $50 \%$ of the black compound has been used in this case, the concentration of the black compound at electrode 2, and thus the voltage, is much lower than when the battery was discharged slowly.

Mathematically, the reduced voltage is therefore described as a function of the discharging current, which can be any function but it is logic to propose the simplest possible, linear relation given in equation 8

$$
U_{d c, t}=U_{d c, t-1}-\alpha \cdot I_{d c, t-1}
$$

Which relates the discharge voltage at time $t$ to the discharge voltage at the previous time interval and a factor $\alpha$ multiplied with the discharging current at time $t-1$. With this equation it is possible to describe the rate capacity effect because a higher discharge current, causes a faster drop of the discharge voltage than a lower current, which is the essence of the rate capacity effect. This equation is applied with success on results of the experiments on the three battery types (see table I) described in figure 1. The values of $\alpha$ for the three battery types are listed in table II, the values in table II were determined on multiple batteries of the same type, and on multiple discharge cycles starting at the maximum SoC, discharging with various currents, on each battery.

TABLE II: $\alpha$ values for the investigated battery types.

\begin{tabular}{clc}
\hline Battery & \multicolumn{2}{l}{$\alpha\left(10^{-4} \mathrm{~V} / \mathrm{A}\right)$} \\
\hline Pb-acid & 1.79 & $+/-0.22$ \\
Li-ion & 2.67 & $+/-0.39$ \\
Seasalt & 2.53 & $+/-0.51$ \\
\hline
\end{tabular}

The value of $\alpha$ appears to be constant for various discharge currents in one battery, and for multiple batteries of the same type. It is likely, however, that the value of $\alpha$ is influenced by the open circuit potential, energy content, size and geometry of a battery. In other words for each battery to be included in simulations using the proposed model, two or more measurements on that battery have to be done to determine the value of $\alpha$. It should also be noted that the value of $\alpha$ is likely to be dependent on the degradation (or ageing) of the battery, because the over-all performance of the battery decreases over time [10]. However, the influence of the battery degradation on the value of $\alpha$ has not been investigated.

For the capacity recovery effect there is no analogy between electric and thermal energy storage. Once mixed, the water in a thermal storage does not recover back to higher temperatures.

Figure 3 shows a schematic representation of a battery during the process of recovery.

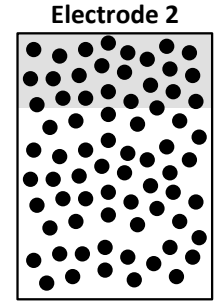

Electrode 1

(a)

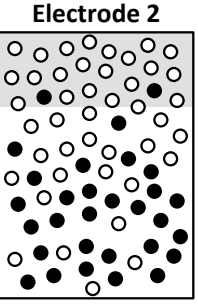

Electrode 1

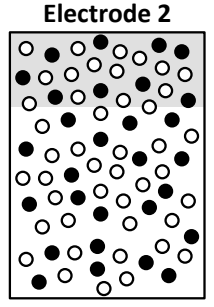

Electrode 1

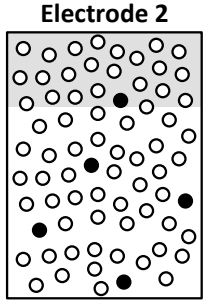

Electrode 1
Fig. 3: Schematic representation of the recovery effect in a battery. a) The battery is fully charged. b) The battery is discharged fast to $50 \%$ of its capacity, but the voltage has dropped to the minimum allowable condition. c) The battery is allowed to rest for a period of time, this results in a redistribution of the black compound. d) The battery is fully discharged.

Figure 3a shows the battery fully charged, and figure $3 \mathrm{~b}$ shows the battery discharged; the concentration of the black compound at electrode 2 is low and thus the voltage is low. Note that the concentration of the black compound near electrode 2 (in the grey area) is lower than the concentration of the black compound elsewhere in the battery. If the battery is then allowed to rest for a period of time (see figure 3c) the black and white compound are properly mixed again, and the 
concentration of black compound in the grey area and thus the voltage is increased. Note that the over-all concentration of the black compound in the battery has not changed during the waiting time. Now the battery can again be discharged (see figure $3 \mathrm{~d}$ ). At the end of the second discharge the concentration of black compound in the grey area and thus the voltage is as low as it was in figure $3 b$ but the over-all concentration of the black compound is lower. Further recovery is not possible.

In figure $4 \mathrm{a}$ it is shown that the recovery effect of the voltage depends on the waiting time. Initially, a first order system approximation for the voltage was assumed with a constant time constant but results were unsatisfactory. When the time constant itself is made a function of the waiting time, the accuracy of the results increases considerably.

As remarked, the recovery effect is described as a first order effect, equation 9.

$$
U_{d c, t^{*}}=U_{d c, t_{0}^{*}}+\left(U_{d c, \max }-U_{d c, t_{0}^{*}}\right) \cdot\left(1-e^{\frac{t^{*}}{\tau_{t}}}\right)
$$

In which $U_{d c, t^{*}}$ the increasing discharge voltage during the waiting time $t^{*}$ from an initial voltage $U_{d c, t_{0}^{*}}$ at the start of the waiting time. $U_{d c, \max }$ is the maximum voltage of the battery at fully charged conditions. $\tau_{t^{*}}$ is the first order time constant which is a function of the waiting time.

For the time constant $\tau$ the following linear relation is introduced which proves sufficient accuracy, equation 10.

$$
\tau_{t^{*}}=\beta \cdot t^{*}+\gamma
$$

It is desired to use an alternative, linear description for the recovery effect given in equation 9 . For this, the exponential function is linearized using the first terms of a Taylor expansion, which yields an approximately equal accuracy, equation 11.

$$
U_{d c, t^{*}}=U_{d c, t_{0}^{*}}+\left(U_{d c, \max }-U_{d c, t_{0}^{*}}\right) \cdot \frac{t^{*}}{\tau_{t^{*}}}
$$

\section{RESULTS}

\section{A. Verification of the model for the Capacity recovery effect}

To verify the method outlined in section II-B predictions made using equations 8 and 11 were compared to the results of discharge experiments on the $\mathrm{Pb}$-acid battery. Figure $4 \mathrm{a}$ shows the battery voltage as a function of time, during two consecutive discharges of the $\mathrm{Pb}$-acid battery and the waiting time between the two consecutive discharges was varied. In this graph points A-E are the end-points of the experiment. For each experiment the minimal state of charge $\left(\mathrm{SoC}_{m i n}\right)$ was determined and summarized in table III.

TABLE III: Experiment settings and measured $\mathrm{SoC}_{\min }$

\begin{tabular}{crrcc}
\hline Experiment & $I_{d c}(\mathrm{~A})$ & $U_{\text {end }}(\mathrm{V})$ & $t_{\text {rest }}(\min )$ & $\mathrm{SoC}_{\min }(-)$ \\
\hline $\mathrm{A}$ & 4 & 5 & - & 0.44 \\
$\mathrm{~B}$ & 1 & 5 & 15 & 0.26 \\
$\mathrm{C}$ & 1 & 5 & 30 & 0.25 \\
$\mathrm{D}$ & 1 & 5 & 60 & 0.23 \\
$\mathrm{E}$ & 1 & 5 & 120 & 0.19 \\
\hline
\end{tabular}

Figure $4 \mathrm{~b}$ shows the predicted $\mathrm{SoC}_{\min }$ for the $\mathrm{Pb}$-acid battery as a function of discharge rate, validated with discharge

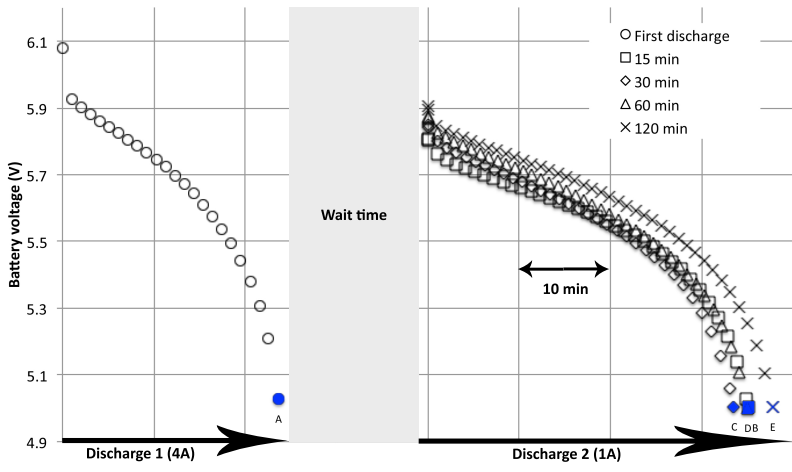

(a) Measured voltage during two consecutive discharge steps, using the $\mathrm{Pb}$-acid battery, with various waiting times. Points A-E indicate the discharge endpoint, for these points the $\mathrm{SoC}_{\min }$ is calculated (see table III)

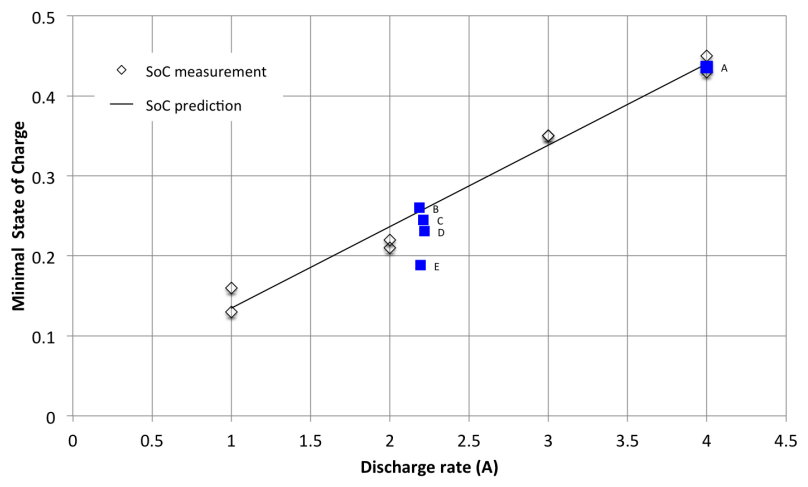

(b) Predicted $\mathrm{SoC}_{\min }$ for the $\mathrm{Pb}$-acid battery, validated with measurements on two $\mathrm{Pb}$-acid batteries. Points A-E indicate the measured $\mathrm{SoC}_{\min }$ for experiments A-E (see table III).

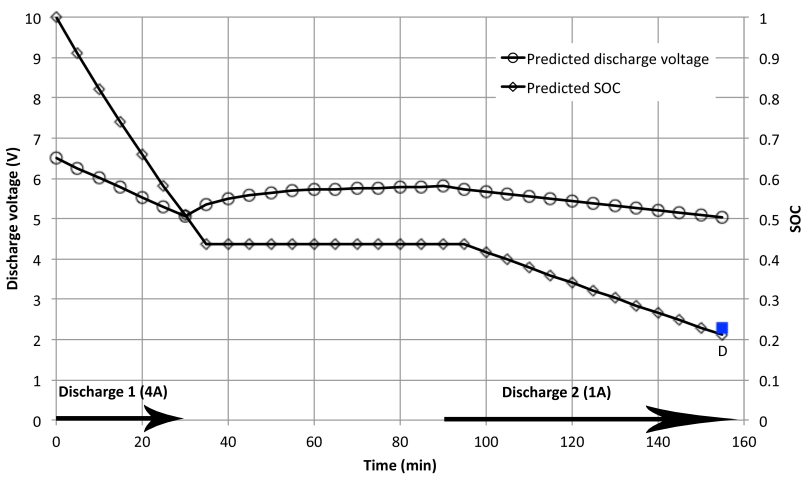

(c) Voltage and $\mathrm{SoC}$, predicted with equation 11 for two consecutive discharge steps on a Pb-acid battery, with a 60 min waiting time (see experiment D in tables III and IV).

Fig. 4: Comparison between predicted and measured voltage and $\mathrm{SoC}$ using the $\mathrm{Pb}$-acid battery.

experiments. The $\mathrm{SoC}_{\min }$ for point $\mathrm{A}$ is predicted correctly, but the $\mathrm{SoC}_{\min }$ of points $\mathrm{B}-\mathrm{E}$ deviate from their predicted values. The deviation from the predicted value seems to be directly related to the waiting time between consecutive discharges. In figure $4 \mathrm{c}$ the discharge current, waiting time, predicted discharge voltage, and predicted $\mathrm{SoC}$, and $\mathrm{SoC}_{\min }$ are shown for experiment $\mathrm{D}$. The discharge voltage is calcu- 
lated with equation 8 during discharging with constant current and with equation 11 during the waiting time period, the appropriate values of $\alpha, \beta$ and $\gamma$ are included in table IV. The SoC is calculated with equations 1, 2 and 3. Similarly the voltage, $\mathrm{SoC}$ and $\mathrm{SoC}_{\min }$ were predicted for experiments $\mathrm{B}, \mathrm{C}$ and $\mathrm{E}$, the results of these predictions are summarized in table IV.

TABLE IV: Simulation settings and results

\begin{tabular}{c|ccc|cc}
\hline Experiment & $\begin{array}{c}\alpha \\
\left(10^{-4} \mathrm{~V} / \mathrm{A}\right)\end{array}$ & $\begin{array}{c}\beta \\
(-)\end{array}$ & $\begin{array}{c}\gamma \\
(\min )\end{array}$ & $\begin{array}{c}\text { SoC }_{\min } \\
\text { Predicted }\end{array}$ & $\begin{array}{c}\mathrm{SoC}_{\min } \\
\text { Measured }\end{array}$ \\
\hline $\mathrm{A}$ & - & - & - & 0.44 & 0.44 \\
$\mathrm{~B}$ & 1.79 & 1.8 & 1.18 & 0.27 & 0.26 \\
$\mathrm{C}$ & 1.79 & 1.8 & 1.18 & 0.23 & 0.25 \\
$\mathrm{D}$ & 1.79 & 1.8 & 1.18 & 0.21 & 0.23 \\
$\mathrm{E}$ & 1.79 & 1.8 & 1.18 & 0.18 & 0.19 \\
\hline
\end{tabular}

In each case the accuracy of the value for $\mathrm{SoC}_{\min }$, is excellent. Besides that, the linear drop of discharge voltage and exponential increase with increasing time constant during the waiting time is realistic. The only drawback of using equation 11 in practice, is that it is necessary, for the determination of the relation between the time constant and the waiting time, to measure the discharge voltage curve of one battery for at least three different waiting times, to determine the values of $\beta$ and $\gamma$

\section{B. Verification in a realistic situation}

To determine the applicability of the proposed model for smart grid control applications, a realistic test was done. The lead-acid battery as described in table I was charged and discharged with various currents, starting from various states of charge, mimicking conditions that could occur when using a battery in real life. This measurement was compared to a simulation of the behavior of a lead acid battery under the same conditions using the proposed model.

The behavior of the battery was also simulated using the well established kinetic battery model (KiBaM), first proposed by Manwell and McGowan [7] and clearly explained by Jongerden and Haverkort [5]. The KiBaM model represents a battery as two communicating wells of charge, the bigger of the two wells can fill the smaller one, while the smaller well can be discharged. The rate capacity effect and the capacity recovery effect are represented by equalization effects between the two wells. The KiBaM model requires the determination of three parameters, in addition to a starting SoC and the maximum energy content $\left(\mathrm{E}_{\max }\right)$ of the battery. Applying the model yields directly the state of charge. The main difference in its approach between the proposed model and the KiBaM model is that in the proposed model the battery voltage is calculated, and from the voltage the SoC is determined; the $\mathrm{KiBaM}$ model makes no use of the battery voltage and calculates the SoC directly. The parameters used for the simulation of the battery behavior are listed in table V.
TABLE V: Settings for simulations done using the proposed model and the KiBaM model

\begin{tabular}{lrl|lrl}
\hline Proposed & & & KiBaM & & \\
\hline$\alpha$ & 1.79 & $10^{-4} \mathrm{~V} / \mathrm{A}$ & $\mathrm{C}$ & 15 & $\mathrm{Wh}$ \\
$\beta$ & 1.8 & - & $\mathrm{C}_{\text {start }}$ & 5,9 & $\mathrm{Wh}$ \\
$\gamma$ & 1.18 & $\min$ & $\mathrm{c}$ & 0.248 & - \\
$\mathrm{E}_{\text {max }}$ & 15 & $\mathrm{Wh}$ & $\mathrm{k}$ & 0.094 & - \\
SoC $_{\text {start }}$ & 0.39 & - & SoC $_{\text {start }}$ & 0.39 & - \\
\hline
\end{tabular}

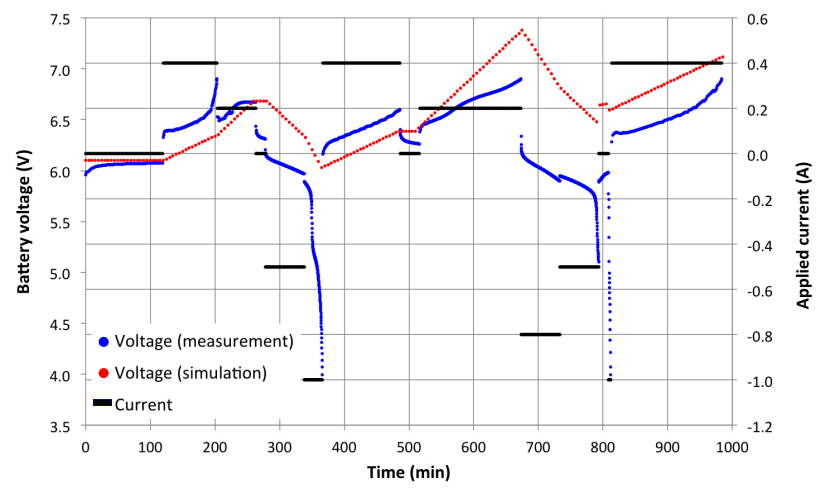

(a) Applied current and resulting voltage of the Pb-acid battery, both measured and calculated with the proposed model. Positive currents represent charging steps, negative currents represent discharging steps.

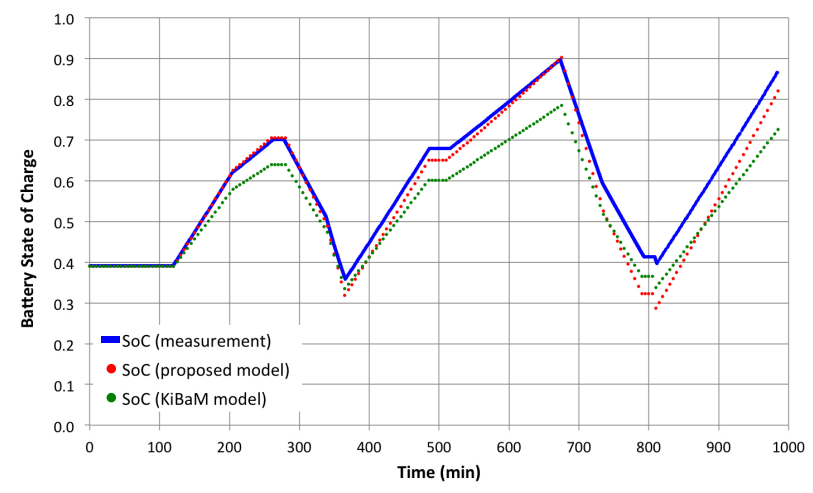

(b) Resulting $\mathrm{SoC}$ of the $\mathrm{Pb}$-acid battery, calculated based on measurements, and simulated with the proposed model and the KiBam model.

Fig. 5: Measurement data and simulation results of the usage of $\mathrm{Pb}$-acid battery in a realistic situation.

The measurement data and the results of the simulations are presented in figure 5. In figure 5a the applied current and resulting / calculated voltage are displayed. The predicted voltage follows roughly the same pattern as the measured voltage. However, the predicted voltage deviates strongly from the measured voltage in the instances where the battery is being discharged deeply, (i.e. discharged to voltages below $5,5 \mathrm{~V})$. This is caused by limitations of the proposed model, the model was designed to predict the parts of the charging and discharging process that are mostly linear with respect to time, whereas the parts where the battery is being deeply discharged are definitely not linear with respect to time. The voltage is also not predicted correctly at instances where the battery starts charging after a period where the battery was not being used (i.e. at $+/-150$ minutes). This occurs because 
there is a difference between the open circuit potential OCP that is measured when the battery is not used, and the charging voltage that is being measured when the battery is charged. The model does not (yet) account for this difference.

However the main purpose of the proposed model is not to give an accurate prediction of the battery voltage over time, but to give an accurate prediction of the $\mathrm{SoC}$ over time. The $\mathrm{SoC}$ over time, calculated from the measured data, and predicted using the proposed model and the $\mathrm{KiBaM}$ model is displayed in figure $5 \mathrm{~b}$. In contrast to the voltage, the $\mathrm{SoC}$ predicted with the proposed model follows the measured SoC closely. In the cases where the predicted SoC deviates from the measured SoC, the predicted $\mathrm{SoC}$ is lower. The deviation from the measured $\mathrm{SoC}$ is under $5 \%$ over all, and about $10 \%$ in the worst case (around 800 minutes). The largest deviations occur at around 350 minutes, and around 800 minutes, these instances coincide with the instances were the battery was deeply discharged, and where the battery voltage was predicted poorly.

The SoC predicted with the KiBaM model also follows the measured SoC closely. Over-all the deviations between the measured SoC and the SoC predicted with the KiBaM model are between $5 \%$ and $10 \%$. In the instances where the SoC predicted with the proposed model showed the largest deviations (i.e. the low points in the graph, around 350 and 800 minutes), the deviations between the measured SoC and the $\mathrm{SoC}$ predicted with the $\mathrm{KiBaM}$ model are noticeably smaller. However, the largest deviations occur as the battery reaches a higher $\mathrm{SoC}$, around 250 and 650 minutes, at these times the proposed model shows little or no deviations. A possible explanation for these deviations is that the $\mathrm{KiBaM}$ model is less suitable to predict the $\mathrm{SoC}$ of batteries that have suffered degradation. To account for battery degradation in the proposed model, one can change the $\mathrm{E}_{\max }$ to reflect the reduced maximum energy content of the degraded battery.

\section{CONCLUSIONS}

A comprehensive model for battery SoC prediction, largely based on earlier work [1], [9] by the authors of this paper is verified and expanded upon. The model is designed to be both accurate and simple enough to be used as part of smart control, and energy usage simulations. Previously the model was verified with data on lead-acid batteries, charged and discharged with constant currents.

Firstly, the model is further verified with measurements on lithium-ion batteries, as well as the experimental seasalt battery. In all experiments on these batteries, presented in figure 1 , the $\mathrm{SoC}$ could be predicted accurately.

Secondly, the model is expanded to accommodate the rate capacity effect and capacity recovery effect. The model is expanded with two linear relations (equations 8 and 11) with which the influence of these two effects on the battery SoC can be predicted. To use these relations in the SoC prediction the parameters $\alpha, \beta$ and $\gamma$ have to be determined from battery discharge experiments; measurements for several discharge currents are required to accurately determine the parameters. The expanded model was verified using measurements on lead- acid batteries, presented in figure 4 . In all instances the SoC of the battery could be accurately predicted using the model. Thirdly, the model is verified in a realistic situation: a lead-acid battery is charged and discharged with various currents, and with varied waiting times between charge and discharge steps. A prediction of the SoC during the same sequence of events is also made using the proposed model. The same prediction is also made using the well-established KiBaM model. The measurements and predictions are displayed in figure 5. The voltage predicted with the proposed model follows the measured voltage only roughly, however, the predicted SoC closely follows the measured SoC, deviating less then 5\% over all.

It is demonstrated that the proposed model is both accurate and easy to use; four measurements on a particular battery are required to predict the battery behavior accurately.

\section{FUTURE WORK}

Future work is dedicated to investigate application of the expanded model for more battery types, including the experimental Seasalt battery and to compare more thoroughly with the KiBam approximation. Another problem to address is to improve the model to take into account the difference between OCP and charge voltage, as discussed in section III-B. Other future work is aimed at integration of the model into the Triana smart grid simulator [11] which is used for simulation studies and as base for embedded smart control systems.

\section{ACKNOWLEDGEMENTS}

The authors thank the Dutch national program TKI Switch2Smartgrids (project Smart Grid Evolution) and the dutch organization RVO for their support.

\section{REFERENCES}

[1] B. Homan, R. P. van Leeuwen, L. Zhu, J. B. de Wit, and G. J. M. Smit, "Validation of a predictive model for smart control of thermal and electrical energy storage," in IEEE Energycon2016.

[2] G. Hoogsteen, A. Molderink, J. L. Hurink, G. J. M. Smit, F. Schuring, and B. Kootstra, "Impact of peak electricity demand in distribution grids: A stress test," in IEEE Powertech 2015.

[3] K. X. Perez, M. Baldea, T. F. Edgar, G. Hoogsteen, R. P. van Leeuwen, T. van der Klauw, B. Homan, J. Fink, and G. J. M. Smit, "Soft-islanding a group of houses through scheduling of chp, pv and storage," in IEEE Energycon 2016.

[4] W.-Y. Chang, "The state of charge estimating methods for battery: a review," ISRN Applied Mathematics, vol. 2013, 2013.

[5] M. R. Jongerden and B. R. Haverkort, "Which battery model to use?" Software, IET, vol. 3, no. 6, pp. 445-457, 2009.

[6] M. Doyle, T. Fuller, and J. Newman, "Modeling of galvanostatic charge and discharge of the lithium/polymer/insertion cell," J. Electrochem. Soc., vol. 140, no. 6, pp. 1526-1533, 1993.

[7] J. F. Manwell and J. G. McGowan, "Lead acid battery storage model for hybrid energy systems," Solar Energy, vol. 50, no. 5, pp. 399-405, 1993.

[8] K. S. Ng, C. S. Moo, Y. P. Chen, and Y. C. Hsieh, "Enhanced coulomb counting method for estimating state-of-charge and state-of-health of lithium-ion batteries," Applied Energy, vol. 86, no. 9, pp. 1506-1511, 2009.

[9] R. van Leeuwen, I. Gebhardt, J. de Wit, and G. Smit, "A predictive model for smart control of a domestic heat pump and thermal storage," in SMARTGREENS 2016.

[10] M. Palacín and A. de Guilbert, "Why do batteries fail?" Science, vol. 315 , no. 6273 , p. $574,2016$.

[11] A. Molderink, "On the tree step methodology for smart grids," Ph.D. dissertation, University of Twente, 2011. 